\title{
EVALUACIÓN DE LA TRANSLATIVIDAD REACTIVA A PARTIR DE LA INTEGRACIÓN DE SECUENCIAS DE PARES DE MODOS LINGÜÍSTICOS REACTIVOS/ACTIVOS ${ }^{1}$
}

\author{
JAIRO TAMAYO*, MONTSERRAT BARRADAS OCAÑA** \\ CENTRO DE ESTUDIOS E INVESTIGACIONES EN CONOCIMIENTO Y APRENDIZAJE HUMANO, \\ UNIVERSIDAD VERACRUZANA, MÉXICO
}

Recibido: 02 de Mayo de 2016

Aprobado: 21 de Mayo de 2016

Para referenciar este artículo:

Tamayo, J. \& Barradas Ocaña, M. (2016). Evaluación de la translatividad reactiva a partir de la integración de secuencias de pares de modos lingüísticos reactivos/activos. Revista Iberoamericana de Psicología: Ciencia y Tecnología, 9 (1), 19-32

\section{Resumen}

\begin{abstract}
La translatividad reactiva ha sido definida como la facilitación secuencial entre dos modos lingüísticos reactivos respecto de su efecto habilitador sobre los modos activos (Ribes-Iñesta, Ibáñez-Bernal y Pérez-Almonacid, 2014). Empleando un procedimiento en el que se evaluó el aprendizaje de la propiedad conmutativa de la suma en niños de quinto grado de educación básica primaria, se analizó la translatividad reactiva a partir de la integración de seis secuencias diferentes de pares de modos lingüísticos reactivos-activos. El objetivo fue observar cuál secuencia afectaba en mayor grado el desempeño de los participantes en la resolución de ejercicios vinculados con la propiedad conmutativa. En el Experimento 1 participaron 18 niños de quinto grado de educación básica primaria, los cuales se dividieron en seis grupos de 3 participantes cada uno. Cada grupo se diferenció por el tipo de secuencia de pares de modos lingüísticos a los que fueron expuestos. En este experimento se emplearon ejercicios de un nivel de complejidad menor (contextual); en el Experimento 2, participaron 18 niños distintos a los anteriores pero del mismo grado escolar y de la misma escuela; se dividieron por grupos y se expusieron a la misma secuencia del Experimento 1. La diferencia radicó en el hecho de que en este caso los ejercicios fueron de un nivel de complejidad mayor (sustitutivo referencial). Las secuencias en las que se presentó la translatividad implicaron a los modos escuchar/hablar-leer/escribir y observar/señalar-leer/escribir. Las principales diferencias se presentaron cuando los ejercicios correspondieron al nivel más complejo.

Palabras clave: aprendizaje comprensivo, translatividad reactiva, modos lingüísticos.
\end{abstract}

\section{ASSESSMENT TRANSLATIVIDAD REACTIVE FROM THE INTEGRATION OF LINGUISTIC PAIR SEQUENCES MODES REACTIVOS / ASSETS}

\begin{abstract}
Reactive translativity has been defined as the sequential facilitation between two reactive linguistics modes regarding their enabling effect on the active linguistic modes (Ribes-Iñesta, Ibáñez-Bernal y Pérez-Almonacid, 2014). This reactive translativity was assessed through a procedure that evaluated learning of the commutative property of addition by fifth-grade children from an elementary school. The reactive translativity was analyzed from the integration of six different sequences of reactive-active pairs of linguistic modes. The goal was to observe which sequence affected more participants' performance when solving exercises related to the commutative property. 18 children from fifth-grade participated in Experiment 1, who were divided in six groups of 3 participants each. Each group was differentiated by the type of sequence of linguistic mode pairs to which it was exposed. The exercises used in this experiment had a lower level of complexity (contextual); 18 different children from the same grade and the same school participated in Experiment 2. They were divided in groups and were exposed to the same sequence as in Experiment 1 . The difference was that for this experiment the exercises used had a higher/greater level of complexity (referential substitute). The
\end{abstract}

1 El estudio reportado forma parte del proyecto número 166345 "Análisis Experimental del Aprendizaje Comprensivo y sus Condiciones", el cual fue financiado por el Consejo Nacional de Ciencia y Tecnología (CONACyT) en el marco de la convocatoria CB-2011-01. Los autores desean agradecer especialmente al Mtro. Fernando Rechy Ramírez por la programación de la tarea experimental empleada en este estudio.

* Doctor en Ciencia del Comportamiento, Profesor e Investigador, Centro de Estudios e Investigaciones en Conocimiento y Aprendizaje Humano, Universidad Veracruzana, México, e-mail: jatamayo@uv.mx

** Licenciada en Psicología, Facultad de Psicología, Universidad Veracruzana, México, e-mail: hypnotic_dream_23@hotmail.com 
sequences in which tranlativity took place were listening / talking-reading / writing and observing / pointing-reading / writing modes. The main differences showed up when using the exercises corresponding to the most complex level. Key words: comprehensive learning, sequential facilitation, linguistic modes..

Los modos lingüísticos corresponden a las diferentes formas en las que el lenguaje puede ocurrir en las interacciones humanas cotidianas. Fuentes y Ribes (2001) clasificaron los modos lingüísticos en reactivos y activos. Su distinción se dio fundamentalmente en términos de la morfología asociada al estímulo o a la respuesta. En los modos reactivos se incluyen observar, leer y escuchar. Se consideran reactivos "en la medida en que no se presentan en modos efectivos sino inefectivos de respuesta y porque además contrastan con la simple audición o visión de eventos naturales." (Pérez-Almonacid, García Utrera y Ortiz Sierra, 2015, p. 94). Cada modo puede, en potencia, generar relaciones funcionales distintas entre el organismo y los eventos con los que entra en contacto. En el caso de los modos lingüísticos activos se encontrarían: señalar o gesticular, escribir y hablar, donde a diferencia de los anteriores, sí ocurren respuestas efectivas. Esta clasificación ha dado lugar a diferentes intentos de identificar experimentalmente el tipo de relaciones que pueden darse entre los modos lingüísticos (e.g. Gómez y Ribes, 2008; Pérez-Almonacid, Pulido y Tamayo, 2015; Pulido y Tamayo, 2015; Tamayo, Ribes y Padilla, 2010; Tamayo y Martínez, 2014; Tamayo y Pulido, 2015; Tamayo y Barradas, 2015).

Una de las relaciones posibles entre modos lingüísticos se ha denominado translatividad activa. Gómez y Ribes (2008) identificaron la translatividad activa a partir del incremento en el desempeño efectivo que ocurre en un modo activo de respuesta como resultado del entrenamiento recibido previamente en otro modo activo. Para evaluarla, emplearon un procedimiento de igualación a la muestra de primer orden en el que presentaron diferentes secuencias de entrenamiento. Cada secuencia varió en términos del orden en el que se requería el modo activo de respuesta. De este modo, una secuencia del tipo escribir-hablar-señalar, implicó que en la primera sesión de entrenamiento, los participantes debían responder al arreglo de estímulos escribiendo el nombre del estímulo que consideraban igualaba correctamente a la muestra; en la segunda sesión de entrenamiento, los participantes ahora debían grabar en voz alta su respuesta; finalmente, en la tercera y última sesión de entrenamiento, ahora se debía responder señalando directamente la figura que se consideraba igualaba a la muestra. Los autores evaluaron todas las secuencias posibles de modos activos y compararon las dos primeras sesiones de entrenamiento a fin de analizar los cambios en términos del número de respuestas correctas que se daban entre una y otra como producto de la manipulación del modo activo de respuesta. Estos cambios se interpretaron como evidencia de la translatividad activa. Los estudios reportados por estos autores evidenciaron que el incremento en el número de respuestas correctas fue mayor cuando en la prueba la respuesta debía darse hablando después de que en el entrenamiento se daba escribiendo. En el mismo sentido, las respuestas correctas incrementaron en la prueba cuando en esta el participante debía dar su respuesta señalando siempre y cuando en el entrenamiento la respuesta se haya dado hablando.

Camacho y Gómez (2007), usaron un procedimiento similar al anteriormente descrito y encontraron que la translatividad ocurrió cuando las respuestas en el entrenamiento implicaron señalar o hablar mientras que en las pruebas se debía escribir o señalar respectivamente. Estos resultados condujeron a plantear que la translatividad activa se daba de los modos más complejos (escribir o hablar) a los menos (señalar). La complejidad se definió en términos del grado de arbitrariedad del sistema convencional vinculado a la morfología de cada modo. El grado de arbitrariedad se definió a partir del sistema convencional articulado al modo y el momento y/o los requerimientos para su aprendizaje durante el desarrollo ontogenético. Por ejemplo, escribir y leer, como productos convencionales humanos se aprenden tardíamente en el desarrollo del individuo, dado que requieren de un programa o ambiente particular de enseñanza, contrario al hablar o al señalar que aparecen de manera previa y "natural" a partir del contacto del niño con sus cuidadores.

Recientemente Ribes-Iñesta, Ibáñez-Bernal y Pérez-Almonacid (2014) desarrollaron una propuesta para estudiar el papel de los modos del lenguaje en el aprendizaje enmarcado dentro de lo que denominaron análisis experimental del aprendizaje comprensivo. Según los autores, las condiciones en las que se puede identificar y evaluar el aprendizaje comprensivo implican atender experimentalmente a dos episodios: un primer episodio en el que el individuo se expone a un conjunto de eventos empleando los modos reactivos lingüísticos (observando, leyendo y/o escuchando) y un segundo episodio en que el individuo responde con morfología lingüística, lo que puede darse en los modos activos o productivos (señala-gesticula, escribe y/o habla). En el segundo episodio, la respuesta dada por el 
individuo en uno u otro modo activo sería el resultado de la forma en que haya entrado en contacto con los eventos por medio del modo reactivo correspondiente.

Ribes-Iñesta et al. (2014) proponen nueve condiciones en las que se puede evaluar el aprendizaje comprensivo a partir de un conjunto de relaciones posibles entre modos reactivos y activos. De las condiciones propuestas, la más evaluada experimentalmente corresponde a la habilitación lingüística. La habilitación se identifica en el desempeño efectivo de los individuos en alguno de los modos activos (e.g. cuando el individuo señala, escribe o habla sobre algo) como resultado de su exposición a eventos con los que se ha relacionado por medio de los modos reactivos (e.g. cuando el individuo observó, leyó o escuchó algo). En esta línea, Pérez-Almonacid, Pulido y Tamayo (2015), evaluaron la habilitación lingüística en el aprendizaje de la propiedad conmutativa de la suma combinando el modo reactivo y activo en el que ocurrían los eventos de estímulo y la respuesta de los participantes respectivamente. Los participantes (niños de quinto grado de primaria) debían observar ejemplares de la propiedad, leer o escuchar sobre la propiedad, para luego resolver ejercicios señalando los ejemplares correctos o incorrectos, completar escribiendo los ejercicios o hacer lo propio hablando. El diseño experimental empleado por los autores estuvo conformado por tres fases: una preprueba, una exposición y una prueba de habilitación. La preprueba buscaba evaluar el grado en que los niños podían resolver ejercicios asociados a la propiedad conmutativa de manera previa a la situación experimental. Por su parte, la prueba evaluaba el efecto de habilitación identificado a partir del incremento de respuestas correctas entre la preprueba y la prueba como resultado de la exposición a ejemplos y ejercicios resueltos durante la exposición, los cuales además requerían la participación de uno u otro modo reactivo. Adicionalmente se emplearon cinco tipos de ejercicios de complejidad creciente, de manera que los participantes en el experimento se distribuyeron al azar en cinco grupos, en función del tipo de ejercicios en los que se entrenaban y debían resolver. Los ejercicios se construyeron de acuerdo con los criterios de logro propuestos por Ribes (2008), a saber: contextual (ligado al objeto, criterio: diferencialidad), suplementario (ligado a la operación, criterio: efectividad), selector (desligado de la operación particular, criterio: precisión), sustitutivo referencial (desligado de la situación presente, criterio: congruencia) y sustitutivo no referencial (desligado de la situación concreta, criterio: coherencia) - ver también Ribes y López (1985) - . Los resultados obtenidos sugirieron que: a) por pares de modos lingüísticos, se presentó una mayor cantidad de casos de habilitación (número de participantes que mejoraron su desempeño en la prueba respecto a la preprueba) en los modos leer/escribir, es decir cuando los participantes resolvieron los ejercicios escribiendo luego de haber leído sobre ellos; b) por nivel de dificultad de los ejercicios, un mayor número de casos de habilitación ocurrió en los niveles de complejidad intermedia y superior (selector y sustitutivo referencial), es decir, al parecer el desempeño mejoró cuando los ejercicios tenían una complejidad intermedia o mayor.

Otra de las condiciones descritas por los autores corresponde a la translatividad reactiva. Esta ha sido definida como "la facilitación secuencial entre dos modos lingüísticos reactivos respecto de su efecto habilitador sobre los modos activos" (Ribes-Iñesta, et al. 2014). En este sentido, este caso sería análogo a la translatividad activa, con la diferencia de que para el caso de la reactiva las secuencias evaluarían el efecto de por lo menos dos modos reactivos sobre la respuesta dada en uno o más modos activos. El propósito de este estudio fue el de evaluar la translatividad reactiva en el contexto del aprendizaje de la propiedad conmutativa de la suma en niños de quinto grado de primaria, empleando ejercicios estructurados en dos niveles de complejidad diferencial, siendo estos el nivel contextual (Experimento 1) y el sustitutivo referencial (Experimento 2). De esta manera se pretendió analizar la translatividad reactiva a la luz de dos fuentes de antecedentes experimentales directos: uno, los hallazgos reportados en el análisis de la translatividad activa; y dos, los resultados obtenidos en el análisis de la habilitación lingüística en el contexto del aprendizaje de la propiedad conmutativa de la suma.

Partiendo de lo anterior, la investigación buscó avanzar en el estudio de las diferentes relaciones que se pueden dar entre modos lingüísticos, específicamente en la condición de translatividad reactiva al: a) comparar si los efectos identificados en el análisis de la translatividad activa se presentaban también en la translatividad reactiva; b) comparar los efectos observados en la translatividad reactiva en relación a los obtenidos en el análisis de la habilitación lingüística en el contexto del aprendizaje de la propiedad conmutativa de la suma; y c) observar si los efectos observados se veían modulados por el nivel de complejidad de los ejercicios sobre la propiedad conmutativa de la suma que los participantes debían resolver. 


\section{Método}

\section{Participantes}

En este experimento participaron un total de 36 niños con un rango de edad entre 10 y 12 años, 20 niños y 16 niñas, estudiantes de quinto grado de educación básica de la Escuela Primaria Hugo Topf, del turno matutino, ubicada en la ciudad de Xalapa, estado de Veracruz, México. Los niños fueron seleccionados de forma aleatoria sin seguir ningún criterio para su distribución en los grupos experimentales. Su participación fue requerida en sesiones de una hora diaria con un máximo de 4 días consecutivos. Se emplearon 18 niños en cada experimento, distribuidos en 6 grupos de 3 participantes cada uno (ver Diseño).

\section{Instrumentos}

Se utilizó un programa en ambiente JAVA, que permitió la presentación de las tareas a los niños. Las respuestas de cada niño se registraron automáticamente y se almacenaron en una base de datos. Los datos fueron analizados en Microsoft Excel ${ }^{\circledR}$ y graficados en SPSS $20 \circledR$ para Windows ${ }^{\circledR}$. Se utilizaron laptops de marca HP, y audífonos marca Logytech con micrófono incluido, en caso de los participantes que requirieron escuchar y/o hablar para responder.

Las sesiones experimentales se realizaron en la biblioteca de la escuela y en ocasiones en un salón de clases, en condiciones relativamente aisladas de ruido. El espacio estuvo acondicionado con mesas, sillas, una computadora portátil y audífonos para cada participante.

\section{Diseño}

Tabla 1. Diseño experimental

\begin{tabular}{|c|c|c|c|c|}
\hline \multirow[b]{2}{*}{ Grupo } & \multicolumn{2}{|c|}{ Fase 1: preprueba } & \multicolumn{2}{|c|}{ Fase 2: exposición / prueba } \\
\hline & pre prueba 1 & $\begin{array}{c}\text { pre prueba } \\
2\end{array}$ & exposición 1 - prueba 1 & exposición 2 - prueba 2 \\
\hline 1 & $\mathrm{obs} / \mathrm{s}$ & esch/h & obs-obs/s & esch-esch/h \\
\hline 2 & $\mathrm{obs} / \mathrm{s}$ & $\mathrm{l} / \mathrm{e}$ & obs-obs/s & $\mathrm{H} / \mathrm{e}$ \\
\hline 3 & esch/h & $\mathrm{obs} / \mathrm{s}$ & esch-esch/h & obs-obs/s \\
\hline 4 & esch/h & l-e & esch-esch/h & $\mathrm{H} / \mathrm{e}$ \\
\hline 5 & $\mathrm{l} / \mathrm{e}$ & obs/s & $\mathrm{H} / \mathrm{e}$ & obs-obs/s \\
\hline 6 & $\mathrm{l} / \mathrm{e}$ & esch/h & $\mathrm{H} / \mathrm{e}$ & esch-esch/h \\
\hline
\end{tabular}

Nota: obs= observar; esch=escuchar; l=leer; s=señalar; h=Hablar; e=escribir. El diseño fue el mismo para los dos experimentos. Solo varió entre experimentos el nivel de complejidad de los ejercicios presentados. 
Los experimentos 1 y 2 variaron en función de la complejidad de los ejercicios que se presentaban a los niños. En el Experimento 1 se emplearon los ejercicios de menor complejidad (denominados nivel contextual), mientras en el Experimento 2 los ejercicios fueron de una complejidad mayor (denominados nivel sustitutivo referencial). Las diferencias entre los dos tipos de complejidad en los ejercicios se describen en el procedimiento. Los demás elementos del diseño fueron los mismos para los dos experimentos. Al interior de cada experimento, cada grupo se diferenció en términos de la secuencia de modos reactivos/activos participantes tal como se puede apreciar en la Tabla 1.Cada grupo contó con 3 participantes (atendiendo al tamaño de la muestra, el análisis estadístico en los resultados se realizó empleando estadísticos no paramétricos).

\section{Procedimiento}

Cada niño fue ubicado frente a una computadora portátil al tiempo que se le explicaba que en la pantalla se le indicaría lo que tenía que hacer, pero que podía preguntar en cualquier momento a los experimentadores todo aquello que no le quedará claro. Posteriormente cada participante pasó a la fase de pre-prueba.

\section{Pre-prueba}

En la pantalla de la computadora apareció una instrucción en forma de texto en la que se enunciaba la propiedad conmutativa de la suma y se describía el ejercicio que a continuación debía resolver (un ejemplo de las instrucciones empleadas se presenta en el Anexo 1). Al presionar el botón "Siguiente" aparecía el ejercicio.

Los ejercicios variaron en términos del criterio del nivel de complejidad evaluado. De este modo, en los ejercicios del nivel contextual (Experimento 1), a los participantes se les presentó conjuntos de pares de sumas (de entre 2 y 4 factores cada una). Frente a cada par debían colocar una "palomita" si el par de sumas cumplía con la propiedad conmutativa, o una "cruz" si no se cumplía. De esta manera, los participantes debían responder diferenciando los ejercicios correctos de los incorrectos. Por su parte, en los ejercicios del nivel sustitutivo referencial (Experimento 2) se presentó un ficha en la parte superior de la pantalla (i.e. nueve), una pila de fichas en el centro, y casillas vacías a los costados en las que se debían ubicar las fichas del centro para completar pares de sumas cuyo resultado fuera el mismo que el presentado en la parte superior de la pantalla y que además cumplieran con la propiedad conmutativa.
En este caso, el participante debía ser congruente en la elección de las fichas respecto al resultado presentado en la parte superior de la pantalla pero también en términos de que cada par de sumas debía cumplir con la propiedad conmutativa (ver Anexo 2).

En todos los casos se presentaron dos ejercicios como los descritos en cada nivel. La forma en que se presentaron los ejercicios y el tipo de respuesta varió en función del par de modos no complementarios. Cuando el modo reactivo fue observar aparecieron figuras de fichas con puntos negros similares a las de dominó; si el modo era leer, en lugar de las fichas apareció el nombre del número en forma de texto; si el modo era escuchar, aparecieron botones de audio que al ser presionados reproducían el nombre del número. Por su parte, cuando el modo activo fue señalar la respuesta requerida fue marcar con el cursor o arrastrar fichas a celdas vacías; en el modo hablar, se presentaron botones que al ser presionados permitían grabar la respuesta del participante; finalmente, en el modo escribir aparecían cajas de texto donde el participante podía teclear su respuesta.

\section{Exposición}

En la exposición los ejercicios presentaron las mismas características de los descritos en la pre-prueba. La única diferencia fue que en este caso la computadora resolvió todos y cada uno de los ejercicios y el participante sólo debía observar cómo lo hacía para resolver la tarea después (esto se le indicó en una instrucción en forma de texto). Los ejercicios se presentaron según el modo reactivo correspondiente a cada participante, esto es, se presentaban las fichas, los nombres de los números en forma de texto o los audios. La computadora iba señalando o construyendo los ejemplos cumpliendo con la propiedad conmutativa de la suma.

\section{Prueba}

La prueba fue idéntica a la pre-prueba, salvo que ahora el participante debía resolver los ejercicios en función de lo observado en la exposición. Cabe aclarar que los dos ejercicios presentados, tanto de exposición como de prueba de habilitación, se alternaron. Esto es, se presentó un ejercicio en la exposición que fue resuelto por la computadora; inmediatamente después un ejercicio de prueba el cual debía ser resuelto por el participante, y así con el siguiente ejercicio. 


\section{Resultados}

Se analizó la translatividad reactiva en términos de la mediana de la diferencia entre el porcentaje de respuestas correctas obtenidos en el segundo par de modos menos el porcentaje del primer par únicamente en las pruebas. Posteriormente se presenta el desempeño por cada uno de los grupos en términos de la mediana de los porcentajes de respuestas correctas en las pre pruebas y pruebas para identificar la tendencia del desempeño al interior de cada grupo. Los resultados se diferencian por nivel evaluado.

\section{Experimento 1: Nivel contextual}

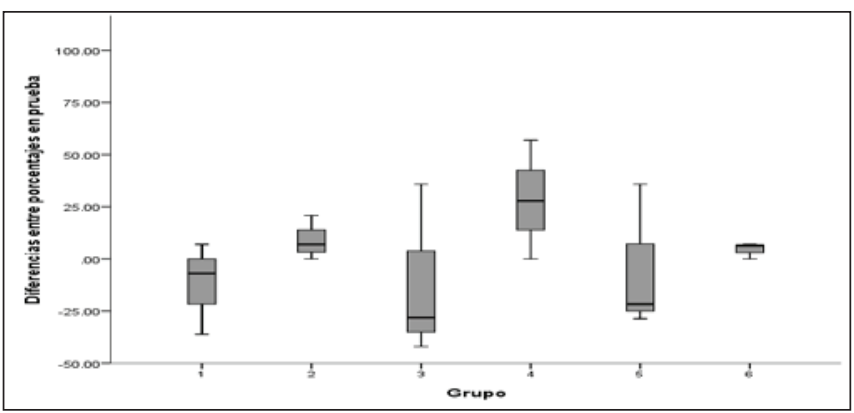

Figura 1: Experimento 1: Nivel contextual
Nota: Mediana y rango de la diferencia entre los porcentajes obtenidos en la prueba en cada uno de los pares de modos de la secuencia en el Experimento 1. El dato se representa por grupos y es un indicador de los casos en los que se presentó translatividad como efecto del desempeño en el primer par sobre el del segundo (diferencia positiva) o no (diferencia igual a 0 ) e interferencia (diferencia negativa).

La mayor diferencia positiva entre los puntajes de la segunda prueba respecto a la primera se presentó en el grupo 4 bajo la secuencia de pares de modos escuchar/ hablar - leer/escribir. Esto indica que el porcentaje de respuestas correctas en la segunda prueba (modos leer/ escribir) fue mayor que el obtenido en la primera prueba (modos escuchar/hablar). En este grupo podría sugerirse la presencia de un efecto de translatividad reactiva.

Por el contrario, los grupos 1, 3 y 5 mostraron diferencias negativas. Esto indica que el porcentaje de respuestas correctas en la segunda prueba fue menor que el obtenido en la primera prueba. Estos resultados pueden sugerir un efecto interferente del desempeño en el primer par de modos respecto al segundo.

Para identificar si las diferencias observadas entre las dos pruebas fueron o no significativas al interior de cada grupo, se aplicó una estadística no paramétrica tipo Wilcoxon. El cálculo no arrojó diferencias significativas al nivel de 0,05.

Figura 2. Mediana del porcentaje de respuestas correctas por grupo obtenidas por los participantes en el Experimento 1.
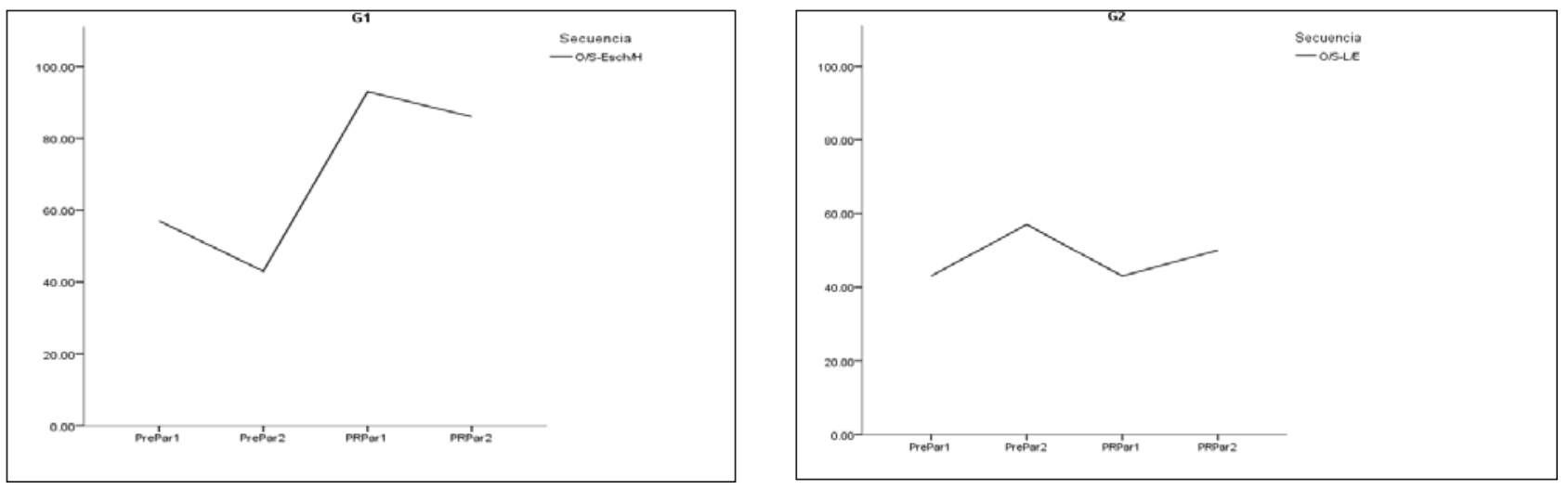

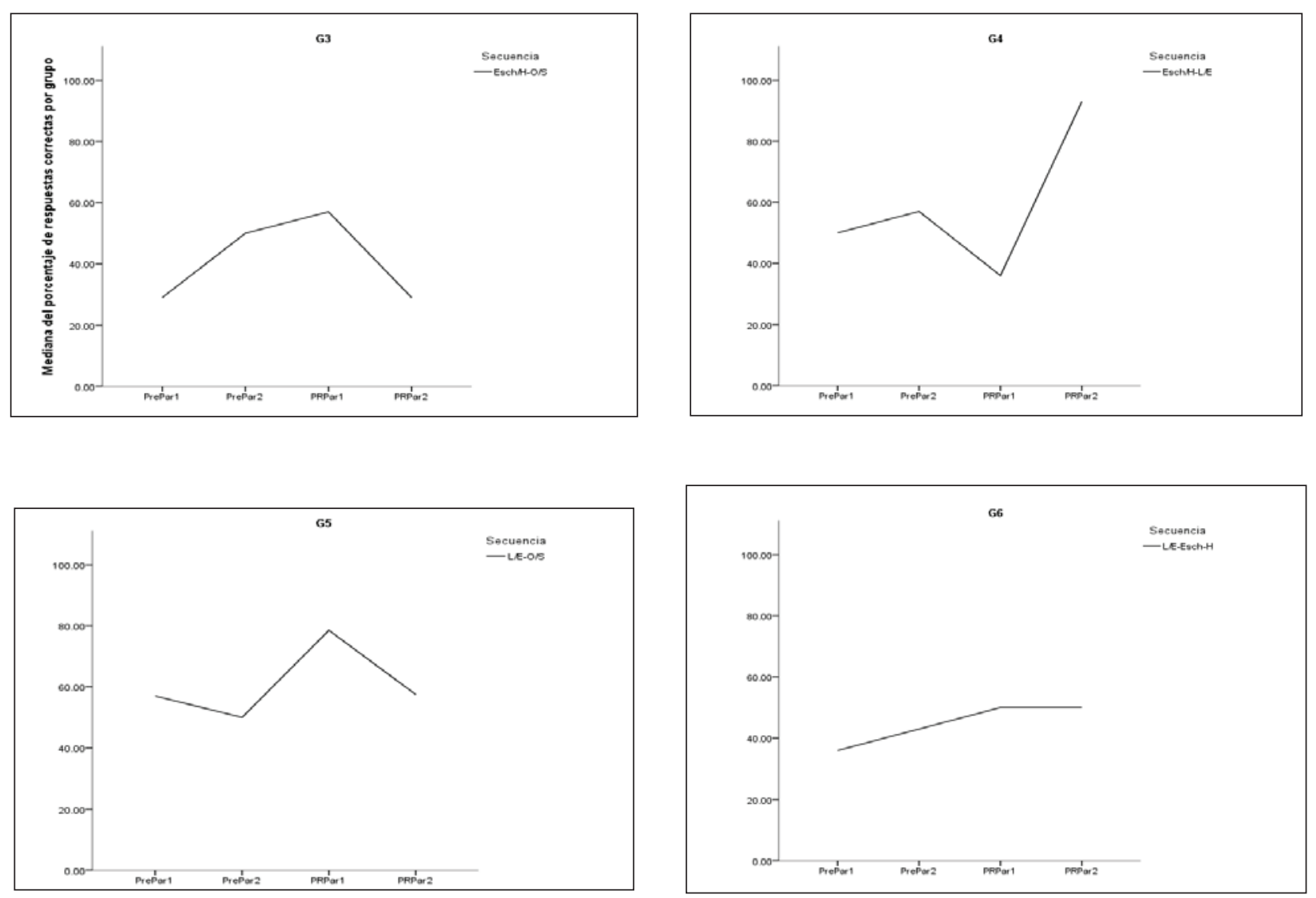

Nota: Se presenta los porcentajes en pre pruebas Precisar (Pre) y pruebas (PR) según la secuencia pares de modos reactivos-activos por grupo. Superior Izquierda, Grupo 1; Superior derecha, Grupo 2; parte media izquierda, Grupo 3; parte media derecha, Grupo 4; parte inferior izquierda, Grupo 5; parte inferior derecha, Grupo 6. O/S: par observar/señalar; L/E: par leer/escribir; Esch/H: para escuchar/hablar.

La tendencia del desempeño por grupos confirma el dato mostrado en la Figura 1. El mayor incremento de la primera prueba a la segunda se presentó en el grupo 4 , mientras que otros presentaron decremento (grupos 1,3 y 5 ). Los otros dos grupos (2 y 6 ) mostraron poca o ninguna diferencia. En general, los mayores cambios entre pre pruebas y pruebas se presentaron en los grupos 1 y 4 . Cabe resaltar que en todos los casos los porcentajes de respuestas correctas en las pre pruebas estuvieron por encima de cero.

\section{Experimento 2: Nivel sustitutivo referencial}

Figura 3: Mediana y rango de la diferencia entre los porcentajes obtenidos en la prueba en cada uno de los pares de modos de la secuencia en el Experimento 2.

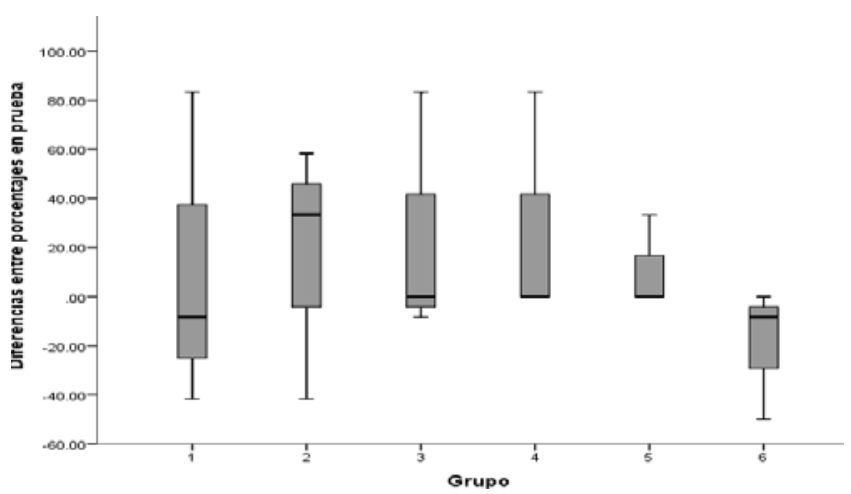

Nota: Las diferencias se calcularon restando el porcentaje obtenido en la prueba en el segundo par, menos el porcentaje obtenido en el primer par de la secuencia. El dato se representa por grupos y es un indicador de los casos en los que se presentó translatividad como efecto del desempeño en el primer par sobre el del segundo (diferencia positiva) o no (diferencia igual a 0) e interferencia (diferencia negativa). 
En el caso del Experimento 2, la mayor diferencia positiva entre las pruebas se observó en el caso del grupo 2 que tuvo la secuencia de pares de modos observar/señalar-leer/escribir. Esto indica que el porcentaje de respuestas correctas fue mayor en la segunda prueba en relación a la primera prueba. A diferencia del Experimento 1, en este caso solo dos grupos ( 1 y 6 ) mostraron una posible interferencia. Los demás grupos mostraron diferencias cercanas al cero sugiriendo la no presencia de un efecto de las secuencias en las pruebas.

Se aplicó un estadístico no paramétrica tipo Wilcoxon para identificar si las diferencias observadas entre las dos pruebas fueron o no significativas al interior de cada grupo. El cálculo no arrojó diferencias significativas al nivel de 0,05. Figura 4. Mediana del porcentaje de respuestas correctas por grupo obtenidas por los participantes en el Experimento 2

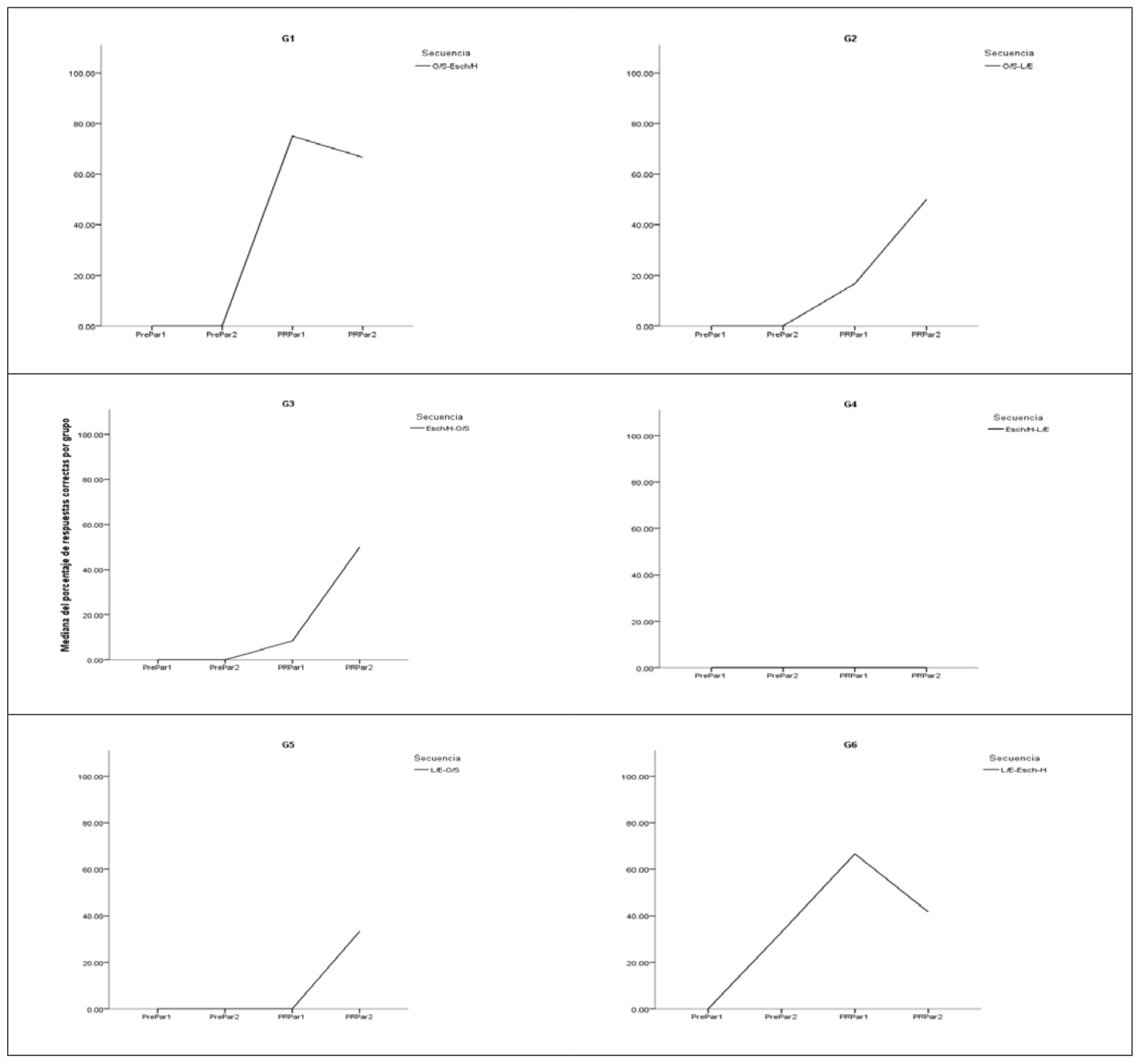

Nota: Se presentan los porcentajes en pre pruebas (Pre) y pruebas (PR) según la secuencia pares de modos reactivos-activos por grupo. Superior Izquierda, Grupo 1; Superior derecha, Grupo 2; parte media izquierda, Grupo 3; parte media derecha, Grupo 4; parte inferior izquierda, Grupo 5; parte inferior derecha, Grupo 6. O/S: par observar/señalar; L/E: par leer/escribir; Esch/H: par escuchar/hablar. 
En términos de la tendencia del desempeño al interior de cada grupo se observó cómo los incrementos en pruebas se presentaron en los grupos 2,3 y 5 , en menor proporción en este último caso. Los grupos 1 y 6 mostraron una disminución de los porcentajes de respuestas correctas entre pruebas, mientras que el grupo 4 no mostró ningún cambio a lo largo de todo el experimento. A excepción de este último caso, en todos los grupos se observó un incremento en el porcentaje de aciertos de las pre pruebas a las pruebas.

Finalmente, a diferencia del Experimento 1, todos los grupos obtuvieron puntajes alrededor del cero en las pre pruebas, lo que hace que el incremento en las pruebas fuera más notorio.

\section{Discusión}

El objetivo del presente estudio fue evaluar el efecto de translatividad reactiva a partir de la integración de secuencias de pares de modos lingüísticos reactivos/activos en el contexto del aprendizaje de la propiedad conmutativa de la suma en niños de quinto grado de educación básica primaria. El efecto se identifica como un incremento en el número de respuestas correctas en el segundo par de la secuencia de modos respecto al mismo criterio de desempeño en el primer par de modos de la misma secuencia. Tal análisis ha permitido comparar si la tendencia del efecto es similar al observado en otros estudios en los que se ha analizado la translatividad activa, si el curso es el mismo al observado en el análisis de la habilitación lingüística en el aprendizaje de la propiedad conmutativa de la suma, y además, si el efecto se modula por la complejidad de los ejercicios que se deben resolver.

Al analizar el efecto de la translatividad reactiva en los dos experimentos se observó que las diferencias no fueron significativas, lo que sugiere que no hubo efecto en términos de las secuencias de pares de modos lingüísticos. A pesar de ello, la representación de los resultados sugiere algunas tendencias distintas en comparación con los hallazgos obtenidos en otros estudios sobre translatividad activa.

Gómez y Ribes (2008) en procedimientos de igualación a la muestra encontraron que la translatividad activa se presentó de los modos activos con morfología más compleja a aquellos con morfología más simple, concretamente del modo escribir al modo hablar y de este último al modo señalar. La complejidad se definió en términos del grado de arbitrariedad del sistema convencional articulado a la morfología de cada modo, y a la necesidad, en su caso, de un programa de enseñanza delimitado y usualmente de carácter escolarizado. Así, la escritura presentaría una morfología más compleja en relación al señalar entre los modos lingüísticos activos. Los resultados encontrados en este estudio sugieren que de haberse presentado el efecto de translatividad reactiva, este pareció seguir un curso opuesto al mostrado en la translatividad activa, esto es, que el traslado pareció darse de los modos de morfología más simple hacia los más complejos, como se observó en los dos experimentos. En el primero, la translatividad se dio entre los pares escuchar/hablar a leer/escribir, mientras que en el segundo, el efecto pudo observarse de los modos observar/señalar a leer escribir. La tendencia opuesta puede verse incluso en las secuencias en que se presentó interferencia, se suponía debería ser en las que se presentara translatividad según los datos observados por Gómez y Ribes (2008).

Sin embargo, al no encontrarse diferencias significativas solo puede sugerirse una tendencia opuesta del efecto respecto a estudios previos. Se requeriría una réplica directa del estudio para evidenciar si la tendencia se mantiene. Es posible que ante dominios distintos al de la matemática, el efecto sea distinto, caso en el cual se asumiría que el dominio delimita el efecto de las secuencias de pares de modos lingüísticos.

Así mismo, los resultados correspondieron parcialmente con los de Pérez-Almonacid, et al. (2015). En el estudio citado se encontró que los niveles en los que se dio mayor habilitación fueron el selector y el sustitutivo referencial; las mayores diferencias también se observaron en el nivel sustitutivo referencial en relación al contextual. Sin embargo, en dicho nivel, la mayor habilitación en el estudio de Pérez-Almonacid et. al. (2015) se presentó cuando los pares fueron leer-escribir, cosa que no sucedió necesariamente en este caso. Las diferencias podrían explicarse por el hecho de que en el estudio en cuestión se evaluaron solamente pares de modos, mientras en este caso se analizaron secuencias de pares de modos lingüísticos. La diferencia entonces puede derivarse de la presentación de las secuencias.

En términos de la tendencia del desempeño al interior de cada uno de los grupos, hay dos hallazgos que merecen especial atención y explicación. El primero de ellos corresponde a que se observaron diferencias en el desempeño en pre prueba entre experimentos. En el Experimento 1, en todos los casos el porcentaje de respuestas correctas en pre prueba fue superior a cero, cosa que no ocurrió en el Experimento 2. El desempeño en pre prueba en el Experimento 1 puede tener dos explicaciones: la primera sugiere que los ejercicios correspondientes al nivel contextual fueron demasiado sencillos, al punto que podían resolverse sin necesidad de conocer sobre la propiedad conmutativa de la suma. Sin embargo, una segunda ex- 
plicación más plausible puede estar en que los ejercicios pudieron haber sido resueltos al azar: en cada grupo de sumas el niño debía decir si este cumplía o no con la propiedad conmutativa, de modo tal que la posibilidad de acertar o errar era del $50 \%$. La mediana del desempeño en todos los grupos (excepción del grupo 3) estuvo entre 40 y 60 , lo que puede estar sugiriendo una alta posibilidad de que los aciertos se hayan dado por azar. Por el contrario, en el caso del Experimento 2, todos los grupos mostraron porcentajes de respuestas correctas cercanas al cero, lo que elimina la posibilidad del azar. Los ejercicios implicaron en este caso la construcción de pares de sumas a partir de un conjunto de valores. Los pares de sumas debían cumplir la propiedad y además sumar un resultado determinado. Indica por lo tanto que para resolver correctamente los ejercicios el niño debía efectivamente aprender sobre la propiedad conmutativa. En este sentido, los incrementos en los porcentajes de respuestas correctas observados en las pruebas del Experimento 2 pueden atribuirse con mayor certeza al efecto de la exposición en que la computadora demostraba al niño la forma correcta de construir las sumas ejemplificando la propiedad conmutativa. Puede concluirse que los ejercicios estructurados en el nivel más complejo (sustitutivo referencial) fueron más sensibles a la manipulación experimental en comparación con los del nivel más simple (contextual). Un ejercicio interesante sería analizar si, en un dominio matemático como el evaluado, una vez alcanzado un desempeño efectivo en el nivel más complejo, aquel se generaliza hacia los niveles más simples sin necesidad de entrenamiento explícito en estos últimos.

El segundo hallazgo interesante corresponde al desempeño general del grupo 4 en el Experimento 2. En este caso, los porcentajes de respuestas correctas fueron cercanos al cero durante todo el experimento. Este resultado podría explicarse en términos del primer modo reactivo en que se dio el contacto con la tarea, que para el caso de este grupo correspondió al modo escuchar. Otros estudios han mostrado un menor efecto de habilitación o facilitación del desempeño cuando el contacto con los eventos sobre los que se debe aprender se da escuchando (Tamayo, Ribes y Padilla, 2010; Tamayo y Martínez, 2014). En el mismo sentido, Varela, Martínez, Padilla, Ríos, y Jiménez (2004), han encontrado en procedimientos de igualación de la muestra que cuando se presenta un mayor número de instancias en modalidad auditiva, el desempeño en la igualación se ve afectado. Es posible entonces que lo observado en este grupo se explique por un efecto de primacía del contacto con el modo escuchar que afectó el desempeño a lo largo del experimento. Sin embargo, esta conclusión requiere ser matizada, dado que tal efecto no se presentó en el grupo 3, el cual también iniciaba con el modo escuchar; tampoco se presentó en el Experimento 1 en ninguno de los dos grupos (3 y 4). Además del efecto de primacía del escuchar, el bajo desempeño del grupo 4 en el Experimento 2 tendría que explicarse por dos factores adicionales: el modo activo que acompañó al modo escuchar (correspondiente al modo hablar en este caso) y al nivel de complejidad de los ejercicios. En este sentido, el bajo desempeño del grupo en cuestión se explicaría por un efecto combinado del contacto inicial con el escuchar, sumado al modo activo hablar en el que los niños debieron resolver los ejercicios (debían dar las respuestas hablando a partir de lo que escuchaban) y al nivel de complejidad de los mismos.

En general, los resultados sugieren que la translatividad reactiva sigue una tendencia distinta a la observada en la translatividad activa y a otras condiciones vinculadas con el aprendizaje comprensivo.

\section{Referencias}

Camacho, J.A., y Gómez, A.D. (2007) Variación de los modos del lenguaje en la adquisición y transferencia del conocimiento. En J.J Irigoyen, M.Y. Jiménez y K.F Acuña, (Eds.), Enseñanza, aprendizaje y evaluación: Una aproximación a la pedagogía de las ciencias (pp. 105-135). México: Editorial UniSon.

Fuentes, M. T., y Ribes, E. (2001). Un análisis funcional de la comprensión lectora como interacción conductual. Revista Latina de Pensamiento y Lenguaje y Neuropsychología Latina, 9 (2), 181-212.

Gómez, D., y Ribes, E. (2008). Adquisición y transferencia de una discriminación condicional del primer orden en distintos modos del lenguaje. Acta Comportamentalia, 16, 183-209.

Pérez-Almonacid, R., García Utrera, L., y Ortíz Cierra, D.M. (2015). El estudio psicológico de la comprensión. En M.A. Reyes Seáñez, G. Mendoza Meraz \& P. Barrera Valdivia (Eds.), Algunas aportaciones psicológicas y sociológicas a la educación, Vol. II (pp. 77-101). México: Nautilium.

Pérez-Almonacid, R., Pulido, L., y Tamayo, J. (2015). La habilitación lingüística en modos complementarios y no complementarios: un análisis integrador. En M.A. Reyes Seáñez, G. Mendoza Meraz y P. Barrera Valdivia (Eds.), Algunas aportaciones psicológicas y sociológicas a la educación, Vol. II (pp. 103-122). México: Nautilium.

Pulido, L., y Tamayo, J. (2015). Aprendizaje comprensivo: evaluación de la habilitación lingüística en modos no complementarios. Revista Mexicana de Investigación en Psicología, 7 (1), 52- 66.

Ribes, E. (2008). Educación básica, desarrollo psicológico y planeación de competencias. Revista Mexicana de Psicología,25 (2), 193-207. 
Ribes, E., y López, F. (1985). Teoría de la conducta un análisis de campo y paramétrico. México: Trillas.

Ribes-Iñesta, E., Ibáñez-Bernal, C., y Pérez-Almonacid, R. (2014). Una propuesta metodológica para el análisis experimental del aprendizaje comprensivo. Revista Mexicana de Psicología, 31 (2), 100-110.

Tamayo, J., y Martinez, D. (2014). Efecto de habilitación en un procedimiento de igualación a la muestra de segundo orden. Revista Mexicana de Investigación en Psicología, 6 (1), 97-108.

Tamayo, J., y Barradas, O. M. (2015). Evaluación de la habilitación a partir de la combinación o secuencia de modos lingüísticos reactivos y activos. IPyE: Psicología y Educación, 9(17), 1-21.

Tamayo, J., y Pulido, L. (2015). Aprendizaje comprensivo: evaluación de la habilitación lingüística en modos complementarios. Journal of Behavior Health \& Social Issues, 7 (2), 57-66.

Tamayo, J., Ribes, E., y Padilla, M. A. (2010). Análisis de la escritura como modalidad lingüística. Acta Comportamentalia, 18 (1), 87-106.

Varela, J., Martínez, C., Padilla, M.A., Ríos, A., y Jiménez, B. (2004). ¿Primacía visual? Estudio sobre la transferencia basada en la modalidad de estímulo y el modo lingüístico. International Journal of Psychology and Psychological Therapy, 4 (1), 67-91.

\section{Anexo 1}

Ejemplo de las instrucciones empleadas en el Grupo 1 del Experimento 1. Las instrucciones variaron entre grupos en función de los modos lingüísticos involucrados. Entre experimentos variaron en función del tipo de ejercicios. En el Experimento 1 se le pedía al niño que diferenciara las sumas en las que se aplicaba la propiedad de las que no; en el Experimento 2 el niño debía construir pares de sumas en las que en todos los casos se cumpliera la propiedad conmutativa a partir de un resultado y empleando un conjunto de números definido.

\section{PRE PRUEBA EJERCICIO 1 MODO SEÑALAR}

!Bienvenido (a)!

Agradecemos tu participación. A continuación se te presentará una serie de tareas. En cada una de ellas se te explicará detalladamente lo que debes hacer.

Presiona el botón "Siguiente" para continuar.

En estas tareas aprenderás sobre algo muy sencillo de matemáticas. En específico sobre la propiedad conmutativa de la suma. La PROPIEDAD CONMUTATIVA DE LA SUMA significa que el orden en el que se presentan los números no cambia el resultado de la suma.

A continuación observarás un resultado, un tache (incorrecto) y una palomita (correcto) en la parte de superior de la pantalla. Debajo de ese resultado aparecerán siete grupos de sumas con las que se puede conseguir ese resultado. Sin embargo, algunas de esas sumas son ejemplos correctos de la propiedad conmutativa de la suma y otras son ejemplos incorrectos.

Deberás indicar cuáles crees que son los ejemplos correctos de la propiedad conmutativa de la suma, arrastrando la palomita a la casilla que corresponda frente al grupo de sumas en las que se cumple la propiedad. En el caso de los ejemplos incorrectos, deberás arrastrar el tache y colocarlo frente al grupo de sumas que tú consideres que sean ejemplos que no cumplan con la propiedad conmutativa de la suma.

Si en cualquier momento deseas volver a leer esta instrucción, presiona el botón "Instrucciones". Si tienes alguna duda, hazla al encargado. De lo contrario, presiona el botón "Siguiente" para iniciar.

\section{PRE PRUEBA EJERCICIO 2 MODO HABLAR}

Ahora seguirás aprendiendo sobre la propiedad conmutativa de la suma. Te recordamos que la PROPIEDAD CONMUTATIVA DE LA SUMA significa que el orden en el que se presentan los números no cambia el resultado de la suma.

En esta parte la tarea es igual que la anterior solo que la forma como se te presentan las cosas es distinta. Presta atención. A continuación se te presentarán en la pantalla unos íconos con un dibujo de una bocina. Inicialmente deberás dar doble clic con el botón izquierdo del mouse a cada icono de las bocinas donde escucharás la palabra igual, un resultado, la palabra incorrecto y la palabra correcto de la parte superior de la pantalla. No avances hasta que hayas escuchado el resultado, la palabra incorrecto y la palabra correcto. Deberás hacerlo de izquierda a derecha.

Debajo de ese resultado aparecerán siete recuadros de sumas con las que se puede conseguir ese resultado. Sin embargo, algunas de esas sumas son ejemplos correctos de la propiedad conmutativa de la suma y otras son ejemplos incorrectos. Da doble clic con el mouse en cada icono para ir escuchando cada uno de los números.

Frente a cada grupo de sumas aparece un botón de color rojo. Debes presionarlo y decir en voz alta la palabra "correcto" si crees que en el grupo de sumas se aplica la propiedad conmutativa de la suma. Si crees que no se aplica la propiedad, presiona el botón y di "Incorrecto". Después de decir la palabra en voz alta vuelve a presionar el mismo botón rojo para que la grabación se detenga. Al lado del botón rojo hay un botón azul. Al presionarlo podrás escuchar lo que grabaste. Si crees que te equivocaste en la respuesta que grabaste, puedes volver a grabarla presionando nuevamente el botón rojo así como lo hiciste 
la primera vez. Después de que hayas respondido todo, podrás continuar haciendo clic en el botón "Siguiente".

Si en cualquier momento deseas volver a leer esta instrucción, presiona el botón "Instrucciones". Si tienes alguna duda, hazla al encargado. De lo contrario, presiona el botón "Siguiente" para iniciar.

\section{EXPOSICIÓN 1 MODO OBSERVAR}

!Bienvenido (a)!

Agradecemos tu participación. A continuación se te presentarán unas tareas parecidas a las que ya hiciste pero esta vez es la computadora que las va a resolver. Presta atención a la forma como lo hace.

Presiona el botón "Siguiente" para continuar.

La PROPIEDAD CONMUTATIVA DE LA SUMA significa que el orden en el que se presentan los números no cambia el resultado de la suma.

En este caso NO tendrás que dar ninguna respuesta. La computadora lo va a hacer todo. Solamente debes prestar atención a la forma como la computadora resuelve la tarea para que después tú puedas hacerlo.

Observarás algunos ejemplos correctos de la propiedad conmutativa de la suma y algunos ejemplos incorrectos. En la parte superior de la pantalla observarás un resultado, un tache y una palomita. También observarás ejemplos de sumas que dan ese mismo resultado, pero sólo en algunos casos se cumple la propiedad conmutativa de la suma y en otras no. Para saber en cuáles ejemplos sí se cumple y en cuáles no se cumple, la computadora señalará con una palomita frente a los ejemplos correctos, mientras que frente a los ejemplos incorrectos señalará con un tache.

$\mathrm{Si}$ en cualquier momento deseas volver a leer esta instrucción, presiona el botón "Instrucciones". Si tienes alguna duda, hazla al encargado. De lo contrario, presiona el botón "Siguiente" para iniciar.

\section{PRUEBA EJERCICIO 1 OBSERVAR- SEÑALAR}

Ahora vas a aplicar lo acabas de aprender.

La PROPIEDAD CONMUTATIVA DE LA SUMA significa que el orden en el que se presentan los números no cambia el resultado de la suma.

A continuación observarás un resultado, un tache (incorrecto) y una palomita (correcto) en la parte de superior de la pantalla. Debajo de ese resultado aparecerán siete grupos de sumas con las que se puede conseguir ese resultado. Sin embargo, algunas de esas sumas son ejemplos correctos de la propiedad conmutativa de la suma y otras son ejemplos incorrectos.

Deberás indicar cuáles crees que son los ejemplos correctos de la propiedad conmutativa de la suma, arras- trando la palomita a la casilla que corresponda frente al grupo de sumas en las que se cumple la propiedad. En el caso de los ejemplos incorrectos, deberás arrastrar el tache y colocarlo frente al grupo de sumas que tú consideres que sean ejemplos que no cumplan con la propiedad conmutativa de la suma.

$\mathrm{Si}$ en cualquier momento deseas volver a leer esta instrucción, presiona el botón "Instrucciones". Si tienes alguna duda, hazla al encargado. De lo contrario, presiona el botón "Siguiente" para iniciar.

\section{EXPOSICIÓN 2 MODO ESCUCHAR}

!Bienvenido (a)!

Agradecemos tu participación. A continuación se te presentarán unas tareas parecidas a las anteriores. Es decir en este caso la computadora resolverá las tareas para que después tú apliques lo que aprendiste. Cambiará la forma como se presentan las cosas en la pantalla. Presta atención.

Presiona el botón "Siguiente" para continuar.

La PROPIEDAD CONMUTATIVA DE LA SUMA significa que el orden en el que se presentan los números no cambia el resultado de la suma.

En este caso NO tendrás que dar ninguna respuesta. La computadora lo va a hacer todo. Solamente debes prestar atención a la forma como la computadora resuelve la tarea para que después tú puedas hacerlo.

A continuación se te presentará una serie de audios que se irán reproduciendo. En la parte superior de la pantalla escucharás un resultado, la palabra correcto e incorrecto. También escucharás ejemplos de sumas que dan ese mismo resultado, pero sólo en algunos casos se cumple la propiedad conmutativa de la suma. Para saber en cuáles ejemplos sí se cumple y en cuáles no se cumple, la computadora dirá la palabra "correcto" después de que hayas escuchado las sumas, mientras que cuando en las sumas no se cumpla la propiedad, la computadora dirá la palabra "incorrecto". Si deseas volver a escuchar los audios deberás dar doble clic con el botón izquierdo del mouse después de que la computadora haya terminado de explicarte todo el ejercicio. Para volver a escuchar los audios deberás dar doble clic en los iconos con el dibujo de las bocinas.

$\mathrm{Si}$ en cualquier momento deseas volver a leer esta instrucción, presiona el botón "Instrucciones". Si tienes alguna duda, hazla al encargado. De lo contrario, presiona el botón "Siguiente" para iniciar. 


\section{PRUEBA EJERCICIO 2 MODO HABLAR}

Ahora vas a aplicar lo que acabas de aprender.

La PROPIEDAD CONMUTATIVA DE LA SUMA significa que el orden en el que se presentan los números no cambia el resultado de la suma.

A continuación se te presentarán en la pantalla unos íconos con un dibujo de una bocina. Inicialmente deberás dar doble clic con el botón izquierdo del mouse a cada icono de las bocinas donde escucharás la palabra igual, un resultado, la palabra incorrecto y la palabra correcto de la parte superior de la pantalla. No avances hasta que hayas escuchado el resultado, la palabra incorrecto y la palabra correcto. Deberás hacerlo de izquierda a derecha.

Debajo de ese resultado aparecerán siete recuadros de sumas con las que se puede conseguir ese resultado. Sin embargo, algunas de esas sumas son ejemplos correctos de la propiedad conmutativa de la suma y otras son ejemplos incorrectos. Da doble clic con el mouse en cada icono para ir escuchando cada uno de los números.

Frente a cada grupo de sumas aparece un botón de color rojo. Debes presionarlo y decir en voz alta la palabra "correcto" si crees que en el grupo de sumas se aplica la propiedad conmutativa de la suma. Si crees que no se aplica la propiedad, presiona el botón y di "Incorrecto". Después de decir la palabra en voz alta vuelve a presionar el mismo botón rojo para que la grabación se detenga. Al lado del botón rojo hay un botón azul. Al presionarlo podrás escuchar lo que grabaste. Si crees que te equivocaste en la respuesta que grabaste, puedes volver a grabarla presionando nuevamente el botón rojo así como lo hiciste la primera vez. Después de que hayas respondido todo, podrás continuar haciendo clic en el botón "Siguiente".

$\mathrm{Si}$ en cualquier momento deseas volver a leer esta instrucción, presiona el botón "Instrucciones". Si tienes alguna duda, hazla al encargado. De lo contrario, presiona el botón "Siguiente" para iniciar.

\section{Anexo 2}

Ejemplo de la forma en la que se presentaban los ejercicios en la pantalla de la computadora. Se muestra sólo el caso del par de modos observar-señalar. En el caso de leer-escribir, en lugar de las fichas con puntos, aparecía el nombre del número (e.g. 6) mientras que los espacios de respuesta aparecían como cajas de texto en las que se podía escribir; en el caso del par escuchar-hablar, en lugar de las fichas aparecieron íconos con bocinas que al ser presionados reproducían el nombre del número mientras que la respuesta debía ser grabada por el participante usando los botones habilitados para tal fin en el espacio de las respuestas.

Experimento 1 nivel contextual

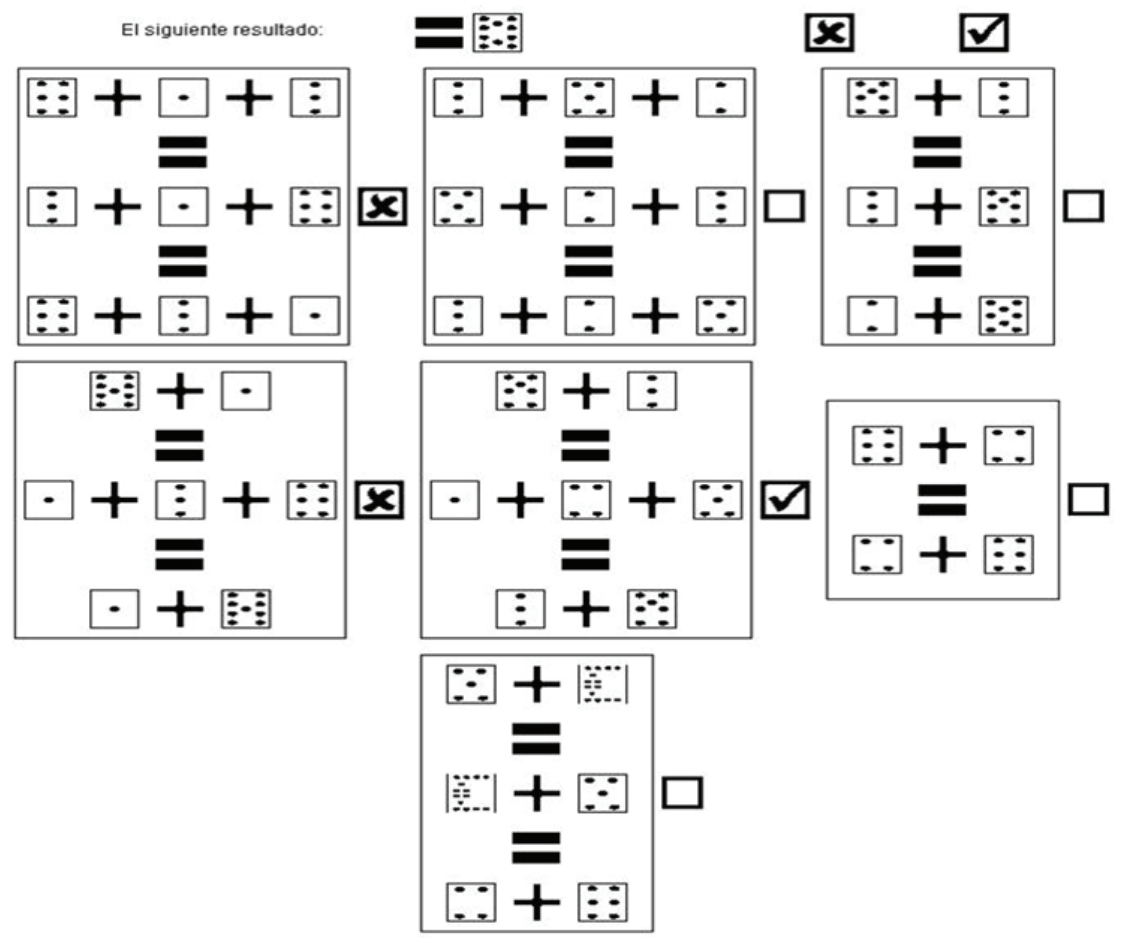


Experimento 2 nivel sustitutivo referencial
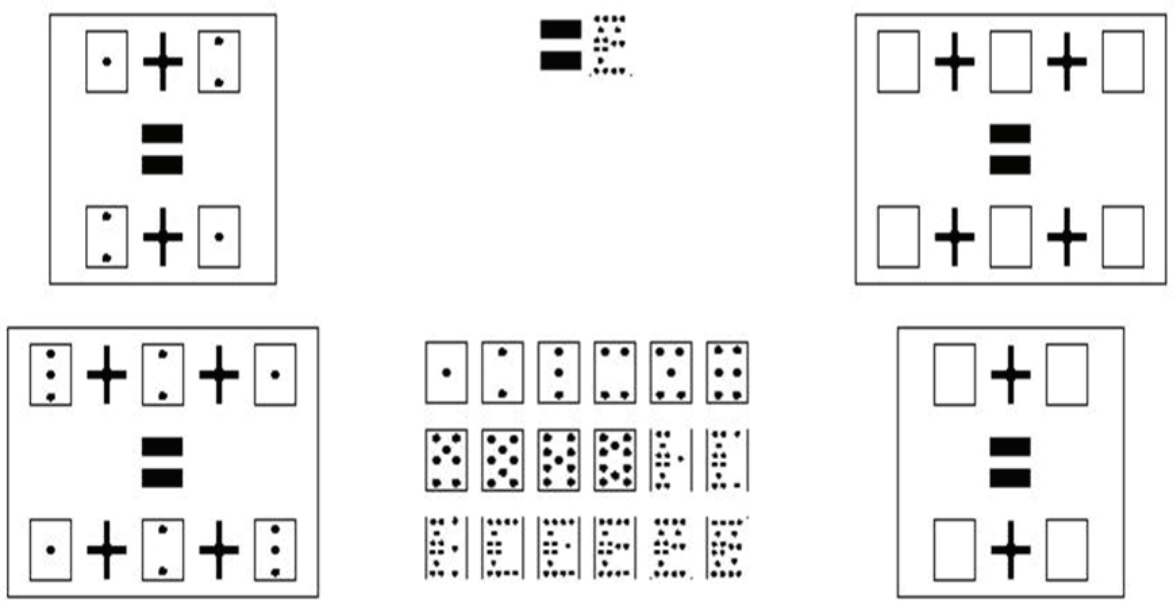

$\square\left[\begin{array}{l}\bullet \\ \bullet\end{array}\right]\left[\begin{array}{l}\bullet \\ \cdots \\ \cdots\end{array}\right]\left[\begin{array}{l}\vdots \\ \vdots\end{array}\right]$

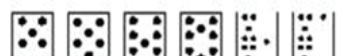

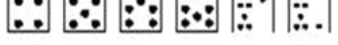

|

|
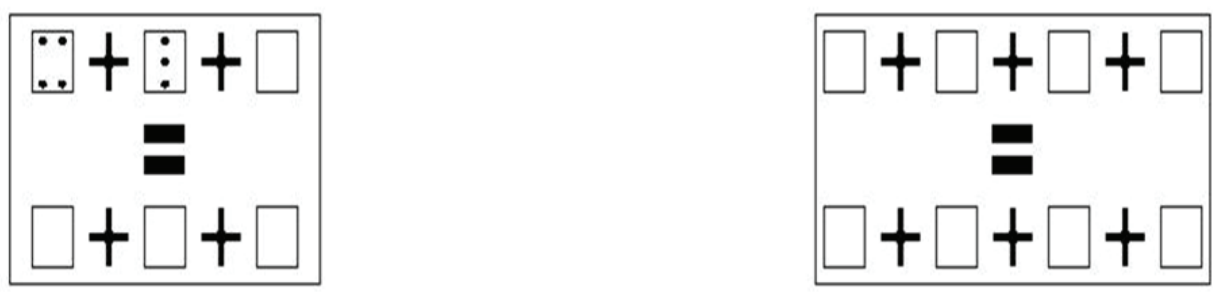